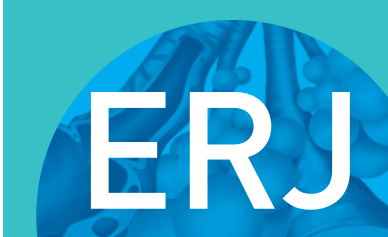

open research

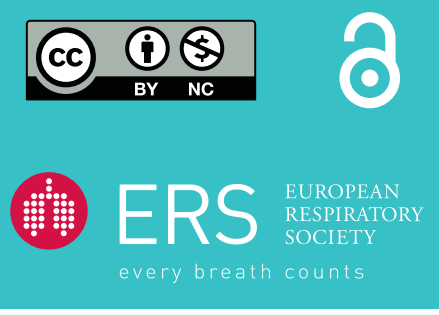

\section{Phagocyte extracellular traps in children with neutrophilic airway inflammation}

\author{
Paul T. King ${ }^{1,2}$, Lovisa Dousha ${ }^{1,2}$, Nadeene Clarke 3,4 , Jennifer Schaefer ${ }^{1,5}$, \\ Rosemary Carzino ${ }^{3,4}$, Roleen Sharma ${ }^{1,2}$, Ken L. Wan ${ }^{6}$, Aveena Anantharajah ${ }^{1,5}$, \\ Kim O'Sullivan², Zhong X. Lu', ${ }^{2,6}$, Stephen R. Holdsworth ${ }^{2,7}$, \\ Sarath Ranganathan ${ }^{3,4,8}$, Philip G. Bardin ${ }^{1,2,9}$ and David S. Armstrong 1,5,10
}

\begin{abstract}
Affiliations: ${ }^{1}$ Monash Lung and Sleep, Monash Medical Centre, Melbourne, Australia. ${ }^{2}$ Monash University Dept of Medicine, Monash Medical Centre, Melbourne, Victoria, Australia. ${ }^{3}$ Paediatric Respiratory Medicine, Royal Children's Hospital, Melbourne, Australia. 'Murdoch Children's Research Institute, Melbourne, Australia. ${ }^{5}$ Monash Children's Hospital, Melbourne, Australia. ${ }^{6}$ Dept of Biochemistry, Monash Pathology, Monash Health, Melbourne, Australia. ${ }^{7}$ Dept of Immunology, Monash Health, Melbourne, Australia. ${ }^{8}$ Dept of Paediatrics, University of Melbourne, Melbourne, Australia. ${ }^{9}$ Hudson Institute of Medical Research, Melbourne, Australia.

${ }^{10}$ Monash University Dept of Paediatrics, Monash Medical Centre, Melbourne, Australia.
\end{abstract}

Correspondence: Paul T. King, Monash Lung and Sleep/Department of Medicine, Monash Medical Centre, 246 Clayton Rd, Clayton, Melbourne, Australia. paul.kinglamonash.edu

ABSTRACT Childhood lung infection is often associated with prominent neutrophilic airway inflammation and excess production of proteases such as neutrophil elastase (NE). The mechanisms responsible for this inflammation are not well understood. One potentially relevant pathway is the production of extracellular traps by neutrophils (NETs) and macrophages (METs). The aim of this study was to measure NET and MET expression in children and the effect of deoxyribonculease (DNase) 1 and $\alpha_{1}$-antitrypsin (AAT) on this process.

We studied 76 children (median age of 4.0 years) with cystic fibrosis or chronic cough who underwent investigational bronchoscopy. NETs, METs and neutrophil elastase activity in bronchoalveolar lavage (BAL) samples were measured using confocal microscopy and functional assays. The effects of DNase 1 and AAT on NET/MET expression and neutrophil elastase activity were examined in vitro.

Both subject groups had airway neutrophilia with prominent BAL production of NETs with neutrophil elastase co-expression; the mean \% $\%$ standard error of the mean of neutrophils expressing NETs in the cystic fibrosis group was $23.3 \pm 2.8 \%$ and in the non-cystic fibrosis group was $28.4 \pm 3.9 \%$. NET expression was higher in subjects who had detectable neutrophil elastase activity $(p \leqslant 0.0074)$. The percentage of macrophages expressing METs in the cystic fibrosis group was $10.7 \pm 1.2 \%$ and in the non-cystic fibrosis group was $13.2 \pm 1.9 \%$. DNase 1 decreased NET/MET expression $(\mathrm{p}<0.0001)$, but increased neutrophil elastase activity $(\mathrm{p} \leqslant 0.0137)$. The combination of AAT and DNase 1 reduced neutrophil elastase activity $(p \leqslant 0.0049)$.

We observed prominent extracellular trap formation in symptomatic children with and without cystic fibrosis. This innate inflammatory response was down-regulated by a combination of currently available therapeutics.

@ERSpublications

Prominent extracellular trap formation may be observed in young children with airway inflammation, with and without cystic fibrosis. This innate inflammatory response is downregulated by a combination of currently available therapeutics. https://bit.ly/3bDaWyC

Cite this article as: King PT, Dousha L, Clarke N, et al. Phagocyte extracellular traps in children with neutrophilic airway inflammation. ERJ Open Res 2021; 7: 00883-2020 [https://doi.org/10.1183/ 23120541.00883-2020].

This article has supplementary material available from openres.ersjournals.com

Received: 25 Nov 2020 | Accepted after revision: 16 Feb 2021

Copyright $\odot$ The authors 2021. This version is distributed under the terms of the Creative Commons Attribution NonCommercial Licence 4.0. For commercial reproduction rights and permissions contact permissions@ersnet.org 


\section{Introduction}

An effective immune response to infection is characterised by an initiation phase with activation of immune effector cells/mediators, then a resolution phase. An inadequate initiation may result in progressive infection; whilst an ineffective resolution may result in excessive inflammation and tissue damage.

Early childhood is a crucial period for lung development. This period is also characterised by a high frequency of respiratory infection, which may potentially have an adverse effect on this development. Young children have an immune response to infection that is less effective than adults. There are major logistical issues in the study of lung infection in children particularly in terms of obtaining relevant samples and imaging. Consequently, the pathogenesis of respiratory infection in childhood remains relatively poorly understood, as does optimal treatment pathways.

Cystic fibrosis lung disease is characterised by recurrent lower airway infection and prominent neutrophilic inflammation that begins in the first year of life [1-3]. The persistent airway infection and inflammation causes bronchiectasis and eventually respiratory failure [4]. The presence of protease neutrophil elastase activity in bronchoalveolar (BAL) fluid in children with cystic fibrosis has been shown to be a primary risk factor for the development of bronchiectasis in this context [5].

Childhood respiratory infection may also have long-term effects in non-cystic fibrosis subjects with decreased lung function and increased risk of development of chronic airways disease [6-11]. A chronic wet cough may be a risk factor for the development of bronchiectasis [12].

One potentially relevant inflammatory pathway is phagocytic extracellular trap formation, an innate immune response. Extracellular traps are produced by neutrophils (NETs) and are composed of extracellular DNA with co-localised mediators such as neutrophil elastase [13, 14]. These NETs help protect against infection, but may also damage host tissue [14-17]. More recently it has been recognised that macrophages also express extracellular traps (METs) and we have demonstrated this in the lung $[18,19]$. NETs have been described in the cystic fibrosis lung but this process is not well studied in young children [20]. NETs and METs are dismantled by deoxyribonuclease (DNase) 1 [14]. DNase 1 is available in the form of inhaled dornase alfa, which is used to break down extracellular DNA and improve sputum clearance in subjects with cystic fibrosis [21]. Dornase alfa is currently recommended for children with cystic fibrosis who are six years and older [22]. The activity of neutrophil elastase is reduced by anti-proteases such as alpha-1 antitrypsin (AAT), which is produced by the liver to control protease activity but also may be given exogenously; e.g. to subjects with AAT deficiency and emphysema.

Our hypothesis is that phagocytic extracellular traps may be present in early life and contribute to lung inflammation, both in subjects with and without cystic fibrosis. Importantly the process of NET and MET expression in childhood may represent a new therapeutic target.

\section{Material and methods}

\section{Study populations}

Two paediatric groups with prominent airway neutrophilia were studied; 1) subjects with cystic fibrosis and, 2) children with chronic cough.

We recruited children from two tertiary paediatric referral centres in Melbourne, Australia: Monash Children's Hospital (MCH)/Monash Health and the Royal Children's Hospital (RCH). Subjects at MCH had a clinically indicated bronchoscopy as part of standard clinical care. Children with cystic fibrosis and non-cystic fibrosis airways disease were recruited from Monash. Subjects at the RCH were recruited as part of the Australian Respiratory Early Surveillance Team for Cystic Fibrosis (AREST CF) protocol [5].

This research project was approved by the Monash Health human research ethics committee and as part of the AREST CF protocol by the ethics committee at RCH. Informed, written consent was obtained from the child's parent/legal guardian prior to enrolment.

It was not feasible to obtain BAL samples in asymptomatic children without airway neutrophilia due to ethical concerns. Therefore we also included as a control group, adults with a chronic cough with normal lung function who had had BAL samples taken previously [18].

\section{Clinical samples}

BAL samples were obtained from anaesthetised patients as previously described [5]. Peripheral blood samples were also obtained on the day of the bronchoscopy. 


\section{Assessment of inflammation}

BAL samples were centrifuged to separate cells and supernatant. The BAL supernatants and serum from peripheral blood were frozen at $-80^{\circ} \mathrm{C}$ pending further analysis. Cell differential count and viability was performed on the BAL samples as previously described $[18,19]$.

To assess trap expression, cells were incubated with the prevalent respiratory bacterium non-typeable Haemophilus influenzae (NTHi), NTHi and DNase 1 or unstimulated (baseline) for one hour. Cells were then fixed and permeabilised and stained for relevant antibodies to define the expression of NETs and METs. Confocal microscopy was used to take images and results were analysed as previously described [18, 19, 23].

Biomarker/inflammatory mediator expression was assessed in BAL and serum samples. In BAL levels of neutrophil elastase activity, AAT and interleukin (IL)-6 were measured. The BAL levels of AAT and IL-6 in both paediatric groups were compared with the adult control group. In the serum samples levels of AAT, then C-reactive protein (CRP) and IL- 6 were measured. AAT levels were measured by nephelometric assay in the serum and an ELISA in the BAL.

Initial samples were used for the analysis of lung extracellular trap expression, whilst subsequent samples were used for the assessment of functional assays of macrophage IL-6 production and effect of AAT on neutrophil elastase activity (there was not enough sample to perform both analyses). Biomarker analysis was done on all samples.

\section{Statistics}

Statistical analysis using paired/unpaired testing with parametric or non-parametric analysis or Chi-squared test was performed as appropriate. Results are expressed graphically as mean \pm SEM or median values and interquartile range as appropriate.

More details are available in the Supplementary Methods in the Online Supplement.

\section{Results}

\section{Characteristics of the study participants}

BAL samples were obtained from 76 participants: 38 children with cystic fibrosis and 38 subjects in the non-cystic fibrosis group. The median age in both groups was 4 years and there were no significant differences in baseline clinical characteristics between the two groups. Both groups had prominent airway neutrophilia and a high prevalence $(\geqslant 66 \%)$ of microbial pathogens; approximately half of the subjects had detectable neutrophil elastase activity in the BAL fluid (table 1). Further details of the subjects (including features of the adult control subjects) are listed in the Supplementary Methods and Supplementary Tables 1 and 2 in the Online Supplement.

\section{Phagocyte extracellular trap expression}

Both the cystic fibrosis and the non-cystic fibrosis groups had prominent expression of NETs and METs at baseline (analysed in the first 46 subjects ( 24 cystic fibrosis and 22 non- cystic fibrosis subjects)). Levels of NET expression were two-fold higher than MET expression. The mean \pm SEM \% of neutrophils expressing NETs in the cystic fibrosis group was $23.3 \pm 2.8 \%$ and in the non-cystic fibrosis group was $28.4 \pm 3.9 \%$. The percentage of macrophages expressing METs in the cystic fibrosis group was $10.7 \pm 1.2 \%$ and in the non-cystic fibrosis group was 13.2 \pm 1.9 The addition of NTHi to unstimulated BAL cells significantly upregulated trap expression in both groups $(p \leqslant 0.0431)$, validating the relevance of infection to the production of NETs and METs. Results are shown in figure 1a-f and in Supplementary Figures 1-3.

\section{TABLE 1 Baseline characteristics of groups}

\begin{tabular}{lccc} 
Characteristic & CF-group & Non-CF group & p-value \\
\hline Children n & 38 & 38 & \\
Age years & $4.1(1.8-6.0)$ & $3.7(1.8-6.7)$ & 0.87 \\
Female/male n & $15 / 23$ & $13 / 25$ & 0.81 \\
Detectable neutrophil elastase activity n/total N (\%) & $23 / 38(61)$ & $17 / 38(45)$ & 0.25 \\
Infection n/total N (\%) & $30 / 38(79)$ & $25 / 38(66)$ & 0.30 \\
Neutrophils in BAL \% & $22(11-52)$ & $23(11-39)$ & 0.81 \\
Subjects with elevated BAL neutrophil \%- n/total N (\%) & $29 / 38(76 \%)$ & $29 / 38(76 \%)$ &
\end{tabular}

Data are presented as median (interquartile range), unless otherwise stated. BAL=bronchoalveolar lavage. An elavated percentage of neutrophils in BAL is defined as $>10 \%$. 


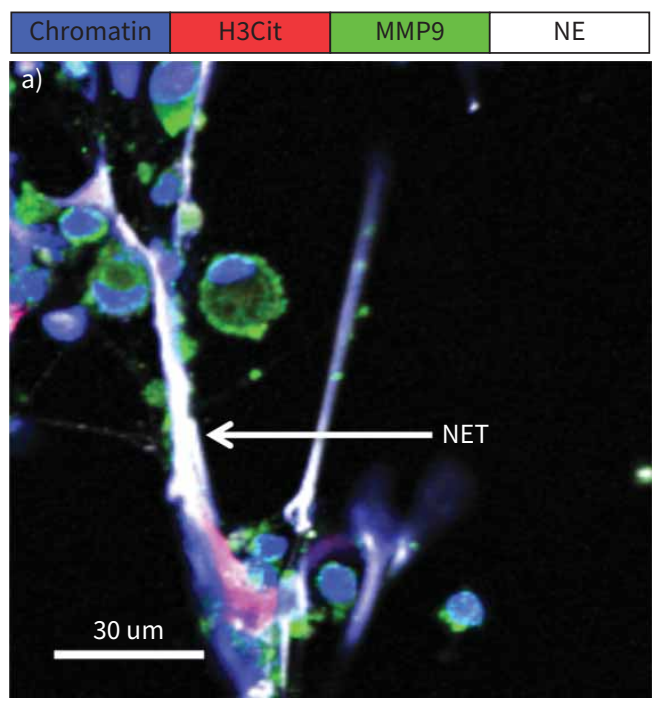

c)

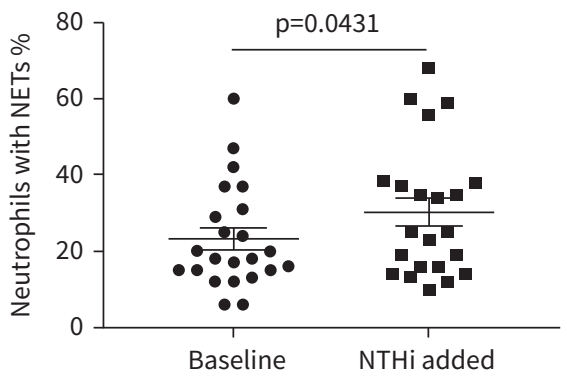

e)

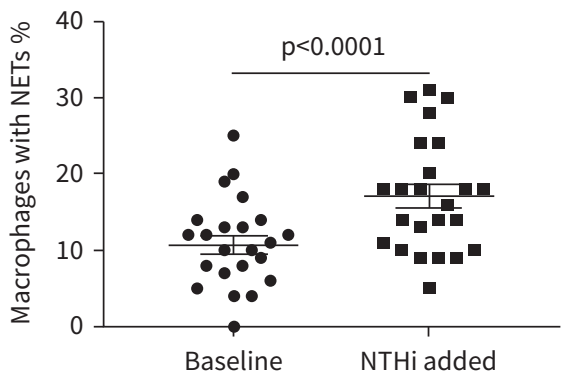

g)

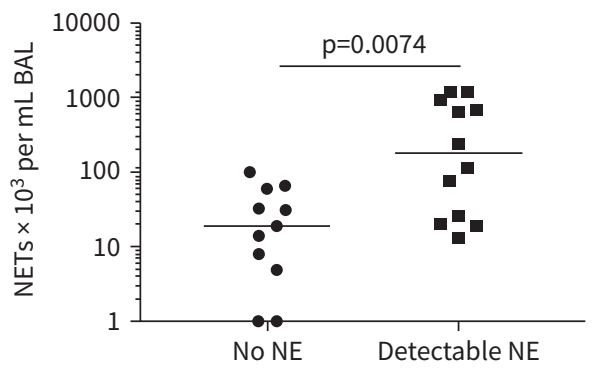

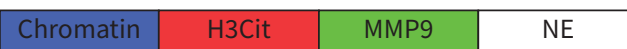

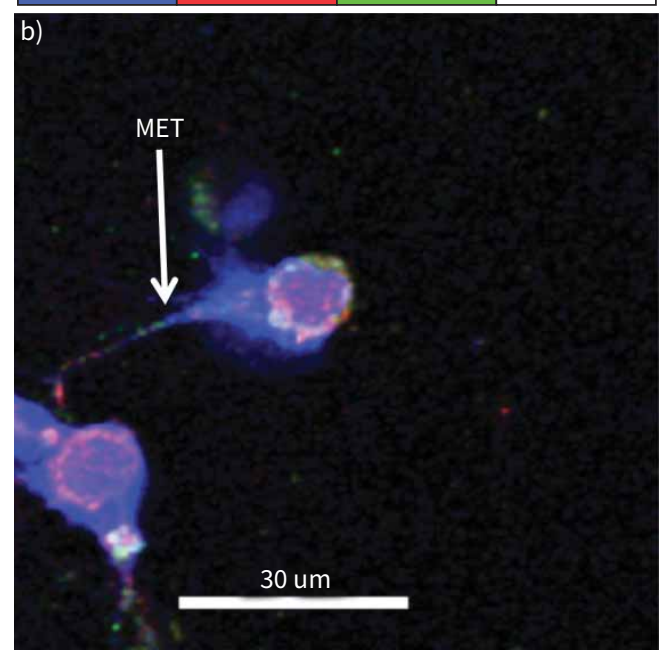

d)

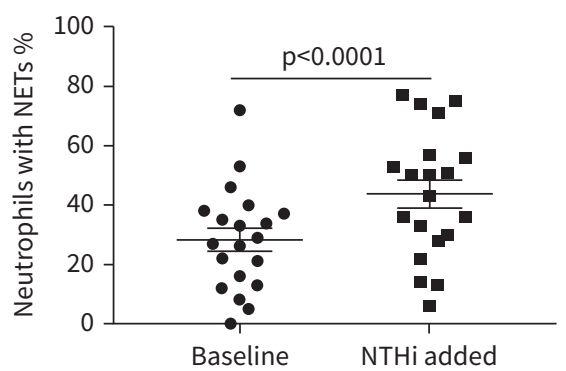

f)

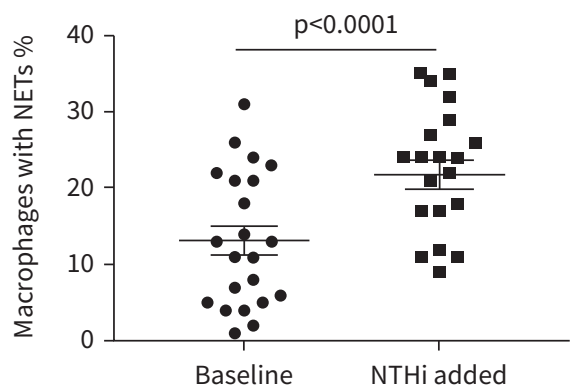

h)

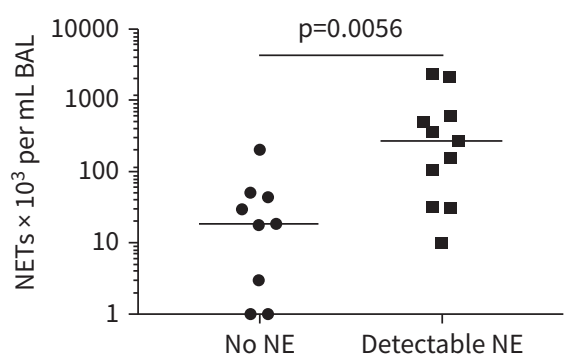

FIGURE 1 Phagocyte extracellular trap expression. Neutrophil (NET) and macrophage (MET) extracellular trap expression was assessed in both the cystic fibrosis (CF) and non-CF groups using confocal microscopy; at baseline and with the addition of the bacterium nontypeable Haemophilus influenzae (NTHi). a) NET expression and b) MET expression. Images shown are merged with chromatin (blue), citrullinated histone (H3Cit) (red), matrix metalloproteinase (MMP) 9 (green) and neutrophil elastase (NE) (white). c) NET expression in CF group and d) NET expression in non-CF group. e) MET expression in CF group and f) MET expression in non-CF group. Bronchoalveolar lavage (BAL) neutrophil elastase (NE) activity was assessed in both g) CF and h) non-CF groups. The number of neutrophils expressing NETs was compared between subjects without detectable NE and with detectable NE. Results shown as mean \pm SEM or median. 


\section{NET expression and neutrophil elastase activity}

An important component of NET formation is the expression of neutrophil elastase; the presence of detectable neutrophil elastase has been shown to be a key risk factor for the development of cystic fibrosis in bronchiectasis. In the cystic fibrosis group the number of neutrophils expressing NETs per mL was compared in subjects with detectable and non-detectable neutrophil elastase activity using an established thresh-hold (figure 1g). The numbers of NETs in subjects with detectable neutrophil elastase was almost 10 -fold higher compared to those subjects with no neutrophil elastase $(\mathrm{p}=0.0074)$.

In the non-cystic fibrosis group similar results were obtained (figure 1h). The numbers of NETs in subjects with detectable neutrophil elastase was 14-fold higher compared to those subjects with no neutrophil elastase $(\mathrm{p}=0.0056)$.

\section{Measurement of IL-6}

Levels of IL-6 an inflammatory cytokine, were measured in the BAL fluid. Nearly all paediatric subjects had detectable IL-6 and levels were comparable between the cystic fibrosis and non-cystic fibrosis groups (figure 2a). BAL macrophages were infected in vitro with NTHi (for $3 \mathrm{~h}$ ), which induced significant upregulation of IL- 6 production in the cystic fibrosis group with a five-fold increase (figure $2 \mathrm{~b}$ ) and the non-cystic fibrosis group with a three-fold increase (figure 2c) $(\mathrm{p}=0.0002)$. Serum IL-6 was generally detectable in both the cystic fibrosis (82\%) and non-cystic fibrosis groups (97\%) with similar levels (figure S5).

\section{Measurement of CRP and AAT levels}

AAT and CRP are acute phase proteins primarily produced in the liver in response to inflammatory signals (e.g. IL-6). Levels of AAT were assessed using an automated nephelometric assay that is used in clinical practice and has a well-established normal range (reference interval). Both groups had median levels that were comparable. Despite these subjects having significant neutrophilic airway inflammation the median values for both groups were in the lower normal range. Both the cystic fibrosis and non-cystic fibrosis populations appeared to be mildly shifted downwards (Figure S6).

In the cystic fibrosis group the serum CRP levels were three-fold higher in subjects with detectable neutrophil elastase compared with subjects with no detectable neutrophil elastase and in the non-cystic fibrosis group six-fold higher (Figure S7a and b) $(p \leqslant 0.041)$. In contrast, levels of serum AAT showed no difference (figure S7c and d).

BAL levels of AAT in both paediatric groups were compared to the adult control group (Table S3). This adult group despite a lack of neutrophilic airway inflammation had levels of AAT that were more than seven-fold higher than the paediatric groups (figure $2 \mathrm{~d}),(\mathrm{p}<0.0001)$.

\section{Effect of DNase1 and AAT}

In both the cystic fibrosis and non-cystic fibrosis groups, the administration of DNase 1 in vitro to NTHi-stimulated cells caused a 20-fold reduction in NET (figure 3a-d) and MET expression (Figure S8) $(\mathrm{p}<0.0001)$.

AAT is the most important anti-protease involved in downregulation of neutrophil elastase activity. The addition of AAT to NTHi-infected blood neutrophils (that expressed NETs) in both groups induced a more than five-fold reduction in neutrophil elastase activity (figure $3 e$ and $f),(p=0.0010)$.

The effect of DNase 1 on NTHi-infected neutrophils was assessed. In contrast to AAT, the addition of DNase 1 increased neutrophil elastase activity approximately two-fold $(\mathrm{p} \leqslant 0.0137)$.

a)

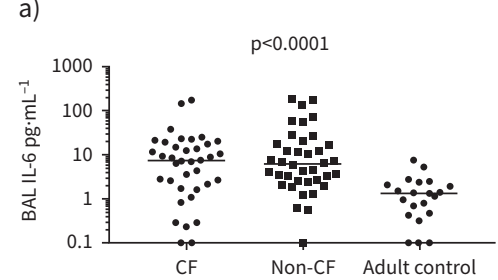

b)

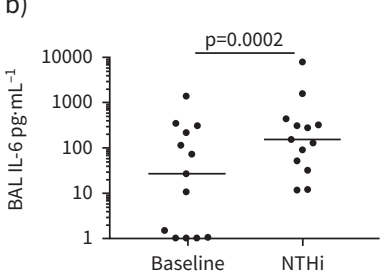

c)

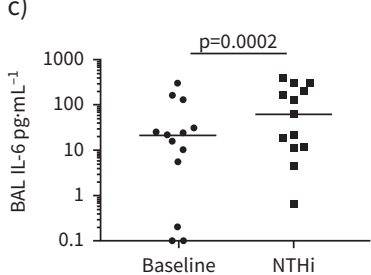

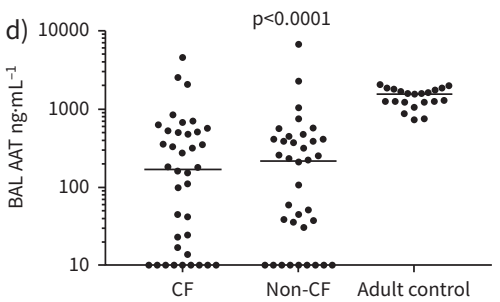

FIGURE 2 Measurement of interleukin (IL) 6 and alpha-1 antitrypsin (AAT) levels. Potential biomarkers/inflammatory mediators were assessed in the BAL fluid. Both childhood groups had detectable BAL IL-6 that was higher than the adult control group (a). BAL macrophage production of IL-6 with nontypeable Haemophilus influenzae (NTHi) infection in cystic fibrosis (CF) (b) and non-CF (c) groups. BAL levels of AAT (measured with ELISA) were compared between CF, non-CF and adult control groups (d). Results expressed as medians. 

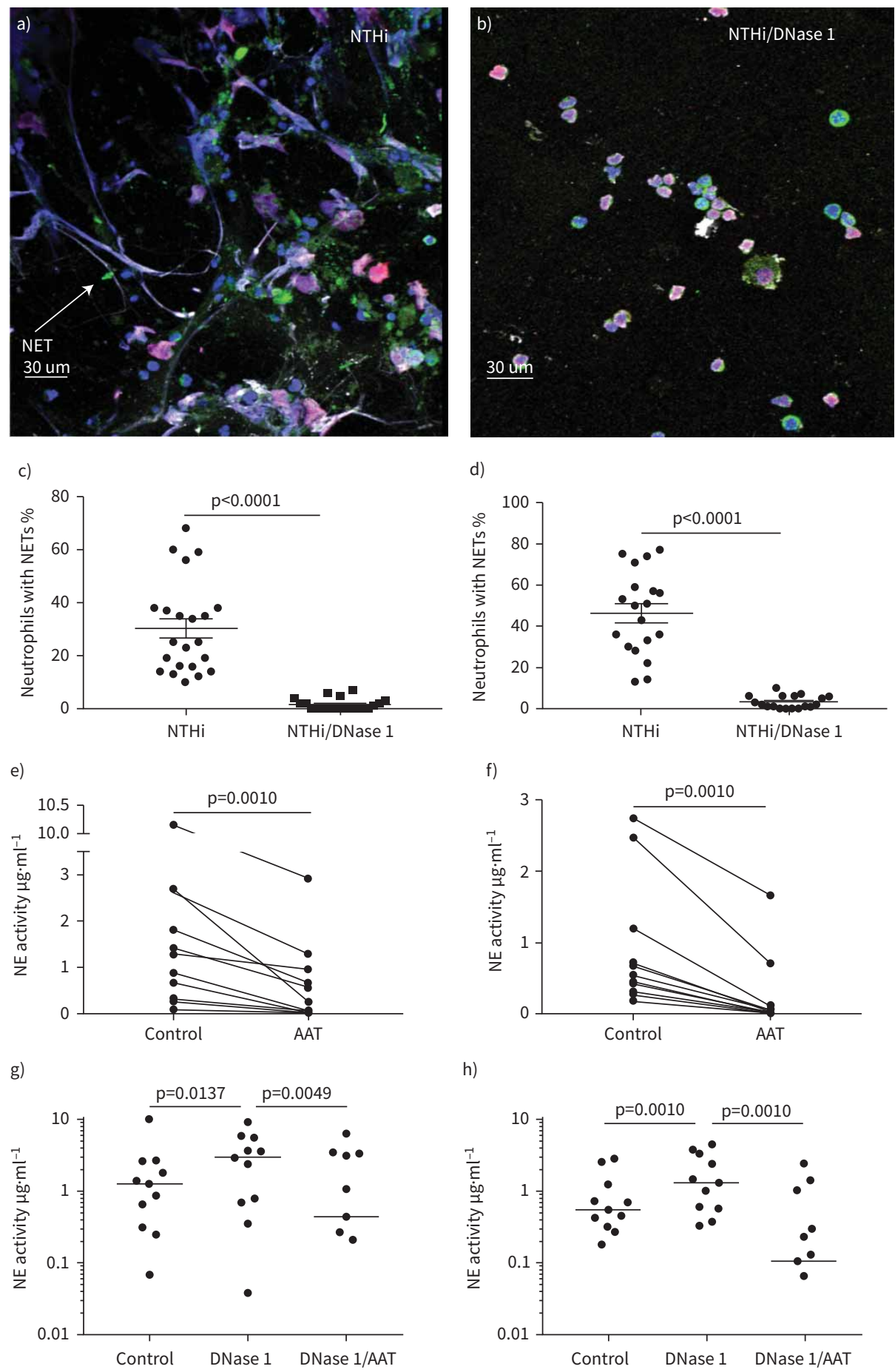

h)
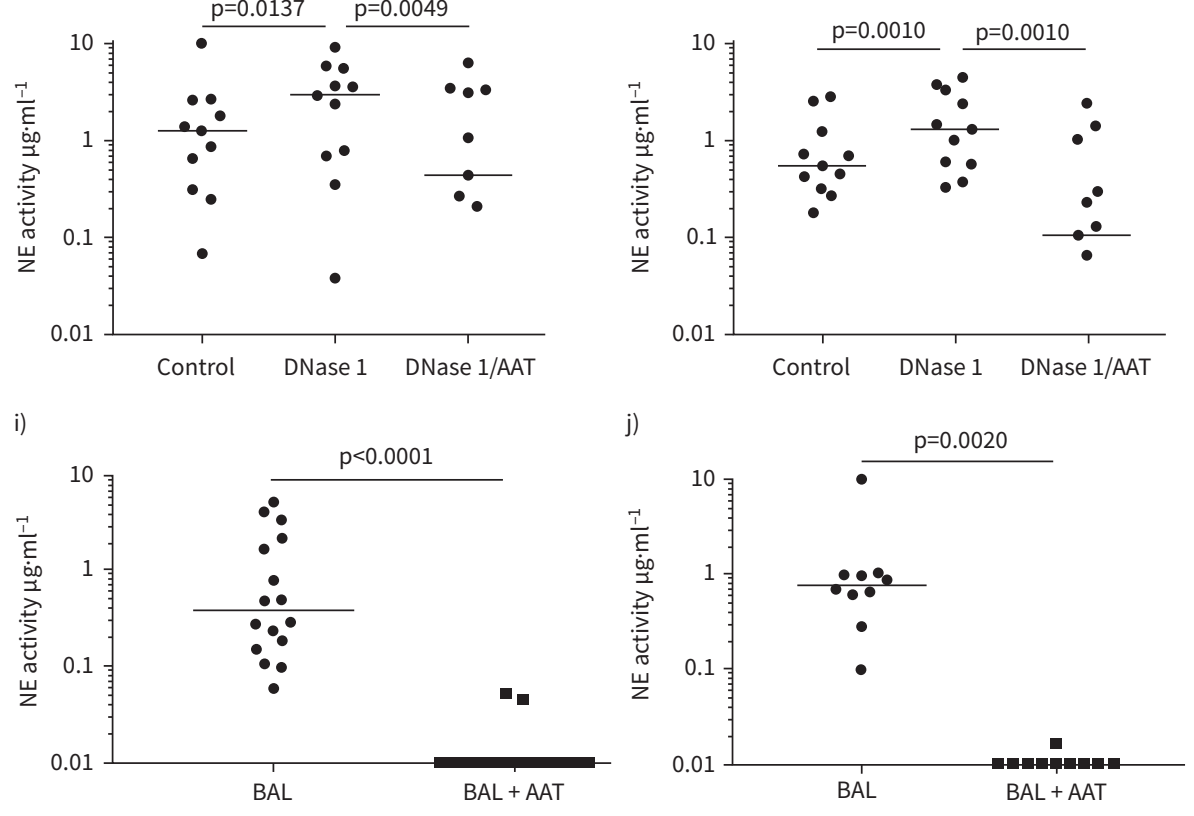
FIGURE 3 Effect of deoxyribonuclease (DNase) 1 and alpha-1 antitrypsin (AAT). The effect of DNase 1 on neutrophil extracellular trap (NET) expression was assessed in both the cystic fibrosis (CF) and non-CF groups. a) Extracellular trap expression in NTHi stimulated cells and b) the effect of the addition of DNase 1. c) NET expression in CF group and d) NET expression in non-CF group. The addition of AAT decreased NE activity compared to control in e) CF subjects and similar results were noted in f) non-CF subjects. DNase 1 increases NE activity which is reduced by the addition of AAT in g) CF subjects and h) non-CF subjects. The addition of AAT to BAL reduces NE activity in i) CF and j) non-CF populations. Results shown as mean \pm SEM or median.

The combination of AAT and DNase 1 was then assessed. This resulted in a six-fold decrease in neutrophil elastase activity in the cystic fibrosis group and a 10 -fold decrease in the non-cystic fibrosis group when compared to DNase 1 alone $(\mathrm{p} \leqslant 0.0049$ ), (figure $3 g$ and $h$ ). Further experiments validated this approach (Figure S9).

The effect of adding AAT on the BAL samples with detectable neutrophil elastase activity was assessed. This resulted in the removal of detectable neutrophil elastase activity in most cases (figure $3 \mathrm{i}$ and $\mathrm{j}$ ).

A summary of the results and their possible relationship to each other is shown in figure 4 .

\section{Discussion}

We have demonstrated prominent neutrophil and macrophage extracellular trap expression in the lungs of young children and have defined pathways involved in their function. Treatment with medications in current clinical use ameliorated trap formation with potential anti-inflammatory effects.

Although NET expression has been described in cystic fibrosis [20], these studies have generally been undertaken in adults and used sputum analysis. There are issues with the use of sputum samples to assess NET expression as there are a variety of causes of cell death that may be responsible for the presence of extracellular DNA in sputum. There is minimal published literature defining the presence of lung NETs in early childhood.

To our knowledge, our work is the largest and most comprehensive study of phagocytic traps in young children. In the current study we have demonstrated the presence of BAL NETs in children with cystic fibrosis from the age of one year onwards. We also studied a non-cystic fibrosis group who were composed of young children with a chronic cough. Similar to the cystic fibrosis group, these children had prominent expression of NETs, which was much higher in those with detectable neutrophil elastase activity. Both

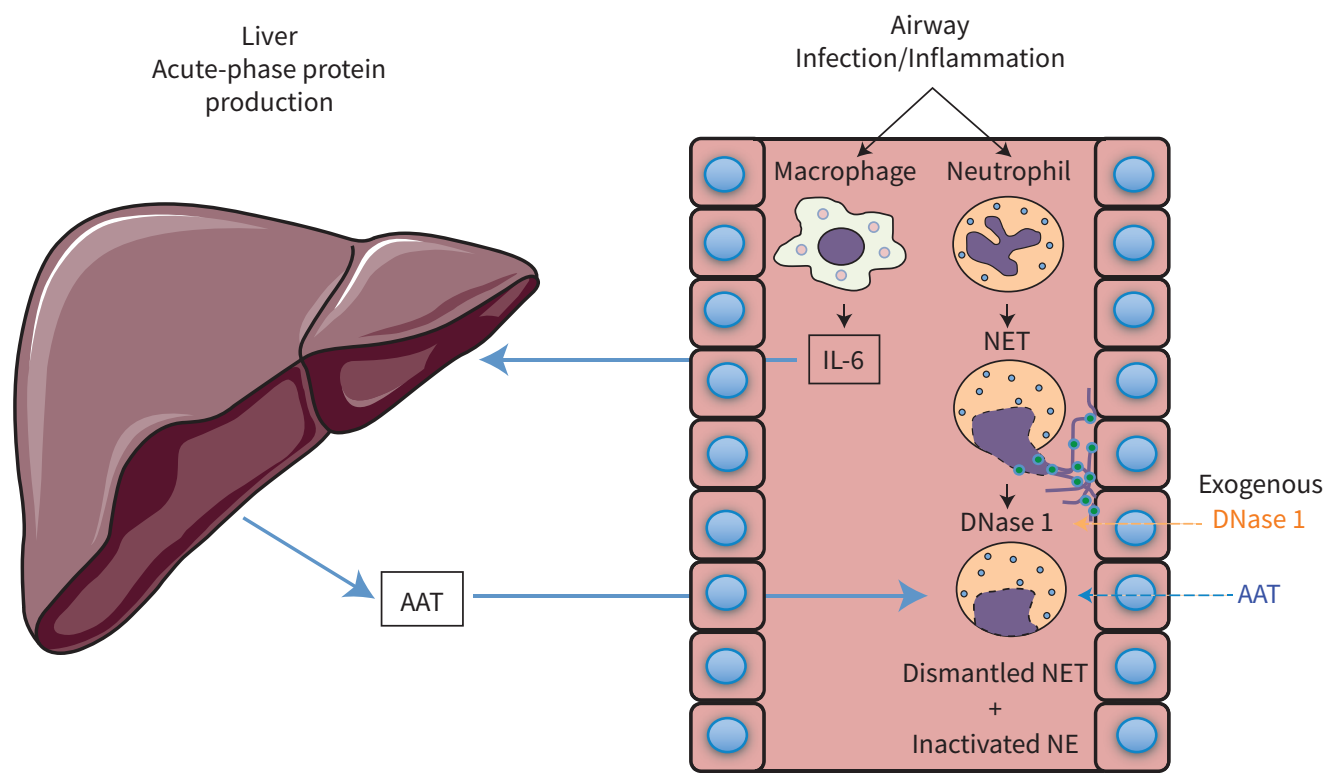

FIGURE 4 Potential relation between key results. Summary of the possible relation between the airway and systemic immune responses. Infection/inflammation in the airway causes the expression of neutrophil extracellular traps (NETs) with neutrophil elastase (NE); as well as activating macrophages to produce inflammatory mediators such as interleukin (IL)-6. One of the potential effects of inflammatory mediators is to act on the liver to induce acute phase reactants such as C-reactive protein (CRP) and $\alpha_{1}$-antitrypsin (AAT). Circulating AAT may then come back to the airway. DNase 1 breaks down NETs and AAT inactivates NE. Both DNase 1 and AAT may be given exogeneously. 
groups of children had marked airway neutrophilia and this was much more prominent than we typically observe in adult subjects.

SLY et al. [5] studied the potential role of a number of factors in the development of bronchiectasis in young children. The key finding of this study was that the presence of detectable neutrophil elastase activity was associated with the development of bronchiectasis. We used the same methodology and demonstrated the levels of NETs (that expressed neutrophil elastase) were much higher in subjects with detectable neutrophil elastase. This result implies that a primary mechanism for the development of lung damage in cystic fibrosis is via NET formation.

In addition to NETs, other forms of extracellular traps have been described such as METs. These METs produce mediators such as matrix metalloproteinase (MMP) 9 and 12, which have shown to have a role in the development of emphysema [24, 25] and potentially also in cystic fibrosis [26-28]. To our knowledge, pulmonary METs have not been described previously in young children, but were present in both groups of subjects and may potentially contribute to host pathology.

Both the cystic fibrosis and non-cystic fibrosis children groups had prominent BAL inflammation with airway neutrophilia. Levels of NETs and to a lesser extent METs were much higher than we have previously observed in adult subjects (for NETs more than 10-fold higher) $[18,19]$. Surprisingly the responses were similar between the two groups. This is likely to be explained by the non-cystic fibrosis group having exacerbated airways disease whilst the cystic fibrosis group were most commonly assessed when at baseline. The results are suggestive that young children make a strong innate immune response in the lung to infection.

Lung inflammation may induce pathways to resolve this response. IL-6 is a key inflammatory cytokine. Both groups had detectable IL-6 in BAL fluid and lung macrophages demonstrated enhanced production with NTHi infection; most subjects also had detectable blood levels of IL-6. Blood levels of IL-6 in young children have not been well established, but the levels were not obviously elevated (as may be expected in inflammatory states). In addition to its inflammatory effects, IL-6 is the primary cytokine that induces the production of acute phase reactants in the lung such as AAT. AAT is predominantly produced in the liver and has a primary role in inactivating proteases, particularly neutrophil elastase. The production of DNase 1 dismantles extracellular trap formation and has an anti-inflammatory effect $[19,29,30]$.

We found that both cystic fibrosis and non-cystic fibrosis children had median serum levels of AAT that were in the lower part of the normal range with both populations being skewed downwards (rather than having outlying subjects). This result is surprising as these children generally had significant airway inflammation (and AAT levels might be expected to be increased). Studies have assessed the role of AAT deficiency alleles in cystic fibrosis; these deficiency alleles have not been found been to be significantly different from normal populations nor were they associated with severity of lung disease [31-33].

Most subjects had detectable AAT in the BAL fluid, but there is very limited literature available regarding what are the normal levels of AAT in the lung. One study described subjects who were healthy smokers as having a median BAL AAT level of $795 \mathrm{ng} \cdot \mathrm{mL}^{-1}$ and in COPD smokers levels were $512 \mathrm{ng} \cdot \mathrm{mL}^{-1}$ (as measured by ELISA) [34]. In the current study we also compared BAL AAT levels in adults with chronic cough with the paediatric groups; levels in children were much lower than adults (in contrast to the IL-6 levels). The results from the current study imply that these children may have a relative deficiency of AAT production in response to inflammation and protease production [35]. The mechanisms responsible for this effect are not clear but could include deficient IL-6 signalling or the inability of young children to upregulate liver AAT production in response to inflammation.

There are several limitations of this study. The first relates to the ethical issue of subjecting asymptomatic children to general anaesthesia to obtain a BAL sample, which required us to use adult controls to compare AAT levels. At our institutions, we do not perform bronchoscopy and BAL in asymptomatic children. Hence we used an adult control group who had chronic cough but did not have airway infection, neutrophilia or NET formation. In our laboratory the normal serum levels of AAT have a range of 1.0 to $2.4 \mathrm{~g} \cdot \mathrm{L}^{-1}$ for the population aged from 1.0 to 70.0 years. Thus serum levels of AAT are roughly comparable between adult and paediatric groups. The number of subjects was moderate in size compared with adult studies, however the results were significant with large differences. The non-cystic fibrosis group was hetereogeneous and whether chronic cough or infection predisposes to the development of lung disease has not been definitively confirmed. The adult controls did not have serum samples available to assess AAT levels.

The results clearly demonstrate that DNase 1 (dornase alfa) reduces NET/MET expression. We have previously demonstrated in vivo in a smoke exposure model that DNase 1 reduces lung proteolysis and similar findings have been described in the liver $[19,36]$. In addition we have demonstrated that this 
therapy markedly reduces lung leukocyte numbers. In other models, DNase 1 has been shown to have a beneficial anti-inflammatory effect $[29,30]$. However DNase 1 was used as a long-term maintenance therapy in a cohort of adults with non-cystic fibrosis bronchiectasis with worse outcomes [37].

Exogenenous AAT has been administered for the treatment of emphysema for a number of years as well as in trials of other lung diseases including cystic fibrosis with inconclusive results [35] DNase 1 has previously been shown to facilitate neutrophil elastase release $[38,39]$. We found similar results in our in vitro NET model. The findings suggest that neutrophil elastase in a trap may be protected from the action of AAT. Our results suggest that before administering DNase 1, assessment of AAT levels is important as this could potentially indicate subjects who are more at risk from tissue damage from released proteases.

The first five years of life are critical for normal lung growth. Our work suggests that both DNase 1 and AAT may have a role in reducing pathogenic lung inflammation in children. Furthermore, our results imply a possible role for the use of combined DNase 1 and AAT, particularly in subjects who may have a relative deficiency of AAT. The potential benefits of this novel approach has to be weighed up against the risks of inhibiting a host immune response and perhaps may be safest to be used for the treatment of exacerbations with co-administered antibiotics. DNase 1 has a short half-life so this medication may need to be given twice rather than once daily. AAT as it is principally derived from human serum, is not widely available in many countries and also is relatively unstable with variable effectiveness as a consequence. This regimen may reduce excessive inflammation not only in cystic fibrosis but also in the much larger non-cystic fibrosis population such as in children with pneumonia.

In conclusion, phagocyte extracellular trap expression is prominent in children, both with cystic fibrosis and non-cystic fibrosis airways disease. This robust innate inflammatory response may potentially contribute to lung damage and represent a new therapeutic target in early life with the possible combination of DNase 1 and AAT.

Acknowledgements: The authors thank Drs Freezer, Roseby and Thielhaber for help with collecting the BALs at Monash. The authors thank the Dept of Clinical Immunology at Monash Health for their assistance. The authors also thank Michael Hickey and Lata Jayaram for critically reviewing the manuscript.

Support statement: This work is supported by grants from the $65 \mathrm{~km}$ for Cystic Fibrosis, the Monash Lung and Sleep Institute and AREST CF. Funding information for this article has been deposited with the Crossref Funder Registry.

Conflict of interest: P.T. King has nothing to disclose. L. Dousha has nothing to disclose. N. Clarke has nothing to disclose. J. Schaefer has nothing to disclose. R. Carzino has nothing to disclose. R. Sharma has nothing to disclose. K.L. Wan has nothing to disclose. A. Ananthrajah has nothing to disclose. K. O'Sullivan has nothing to disclose. Z.X. Lu has nothing to disclose. S.R. Holdsworth has nothing to disclose. S. Ranganathan has nothing to disclose. P.G. Bardin has nothing to disclose. D.S. Armstrong has nothing to disclose.

\section{References}

1 Cantin AM, Hartl D, Konstan MW, et al. Inflammation in cystic fibrosis lung disease: pathogenesis and therapy. J Cyst Fibros 2015; 14: 419-430.

2 Armstrong DS, Hook SM, Jamsen KM, et al. Lower airway inflammation in infants with cystic fibrosis detected by newborn screening. Pediatr Pulmonol 2005; 40: 500-510.

3 Pillarisetti N, Williamson E, Linnane B, et al. Infection, inflammation, and lung function decline in infants with cystic fibrosis. Am J Respir Crit Care Med 2011; 184: 75-81.

4 Nichols DP, Chmiel JF. Inflammation and its genesis in cystic fibrosis. Pediatr Pulmonol 2015; 50 Suppl. 40: S39-S56.

5 Sly PD, Gangell CL, Chen L, et al. Risk factors for bronchiectasis in children with cystic fibrosis. N Engl J Med 2013; 368: 1963-1970.

6 Johnston ID, Strachan DP, Anderson HR. Effect of pneumonia and whooping cough in childhood on adult lung function. N Engl J Med 1998; 338: 581-587.

7 Bui DS, Lodge CJ, Burgess JA, et al. Childhood predictors of lung function trajectories and future COPD risk: a prospective cohort study from the first to the sixth decade of life. Lancet Respir Med 2018; 6: 535-544.

8 Svanes C, Sunyer J, Plana E, et al. Early life origins of chronic obstructive pulmonary disease. Thorax 2010; 65: $14-20$.

9 Montgomery S, Bahmanyar S, Brus O, et al. Respiratory infections in preterm infants and subsequent asthma: a cohort study. BMJ Open 2013; 3: e004034.

10 Perret JL, Lodge CJ, Lowe AJ, et al. Childhood pneumonia, pleurisy and lung function: a cohort study from the first to sixth decade of life. Thorax 2020; 75: 28-37.

11 Chan JY, Stern DA, Guerra S, et al. Pneumonia in childhood and impaired lung function in adults: a longitudinal study. Pediatrics 2015; 135: 607-616.

12 Goyal V, Grimwood K, Marchant J, et al. Does failed chronic wet cough response to antibiotics predict bronchiectasis? Arch Dis Child 2014; 99: 522-525.

13 Brinkmann V, Reichard U, Goosmann C, et al. Neutrophil extracellular traps kill bacteria. Science 2004; 303: $1532-1535$.

14 Brinkmann V, Zychlinsky A. Neutrophil extracellular traps: is immunity the second function of chromatin? J Cell Biol 2012; 198: 773-783. 

189. 2689-2695.

6 Porto BN, Stein RT. Neutrophil extracellular traps in pulmonary diseases: too much of a good thing? Frontiers Immunol 2016; 7: 311

17 Cheng OZ, Palaniyar N. NET balancing: a problem in inflammatory lung diseases. Frontiers Immunol 2013 ; 4: 1.

18 King PT, Sharma R, O'Sullivan K, et al. Nontypeable Haemophilus influenzae induces sustained lung oxidative stress and protease expression. PLoS ONE 2015; 10: e0120371.

19 King PT, Sharma R, O'Sullivan KM, et al. Deoxyribonuclease 1 reduces pathogenic effects of cigarette smoke exposure in the lung. Sci Rep 2017; 7: 12128.

20 Khan MA, Ali ZS, Sweezey N, et al. Progression of cystic fibrosis lung disease from childhood to adulthood neutrophils, neutrophil extracellular trap (NET) formation, and NET degradation. Genes (Basel) 2019; $10,183$.

21 Konstan MW, Ratjen F. Effect of dornase alfa on inflammation and lung function: potential role in the early treatment of cystic fibrosis. J Cyst Fibros 2012; 11: 78-83

22 Mogayzel PJ Jr, Naureckas ET, Robinson KA, et al. Cystic fibrosis pulmonary guidelines. Chronic medications for maintenance of lung health. Am J Respir Crit Care Med 2013; 187: 680-689.

23 Sharma R, O'Sullivan KM, Holdsworth SR, et al. Visualizing macrophage extracellular traps using confocal microscopy. J Vis Exp 2017; 128, 56459.

24 Churg A, Zhou S, Wright JL. Series "matrix metalloproteinases in lung health and disease": Matrix metalloproteinases in COPD. Eur Respir J 2012; 39: 197-209.

25 Hautamaki RD, Kobayashi DK, Senior RM, et al. Requirement for macrophage elastase for cigarette smoke-induced emphysema in mice. Science 1997; 277: 2002-2004.

26 Sagel SD, Kapsner RK, Osberg I. Induced sputum matrix metalloproteinase-9 correlates with lung function and airway inflammation in children with cystic fibrosis. Pediatr Pulmonol 2005; 39: 224-232.

27 Garratt LW, Sutanto EN, Ling KM, et al. Matrix metalloproteinase activation by free neutrophil elastase contributes to bronchiectasis progression in early cystic fibrosis. Eur Respir J 2015; 46: 384-394.

28 Devereux G, Steele S, Jagelman T, et al. An observational study of matrix metalloproteinase (MMP)-9 in cystic fibrosis. J Cyst Fibros 2014; 13: 557-563.

29 Merza M, Hartman H, Rahman M, et al. Neutrophil extracellular traps induce trypsin activation, inflammation, and tissue damage in mice with severe acute pancreatitis. Gastroenterology 2015; 149: 1920-1931.

30 Chen G, Zhang D, Fuchs TA, et al. Heme-induced neutrophil extracellular traps contribute to the pathogenesis of sickle cell disease. Blood 2014; 123: 3818-3827.

31 Mahadeva R, Westerbeek RC, Perry DJ, et al. Alpha1-antitrypsin deficiency alleles and the Taq-I G-->A allele in cystic fibrosis lung disease. Eur Respir J 1998; 11: 873-879.

32 Mahadeva R, Stewart S, Bilton D, et al. Alpha-1 antitrypsin deficiency alleles and severe cystic fibrosis lung disease. Thorax 1998; 53: 1022-1024.

33 Frangolias DD, Ruan J, Wilcox PJ, et al. Alpha 1-antitrypsin deficiency alleles in cystic fibrosis lung disease. Am J Respir Cell Mol Biol 2003; 29: 390-396.

34 Ropcke S, Holz O, Lauer G, et al. Repeatability of and relationship between potential COPD biomarkers in bronchoalveolar lavage, bronchial biopsies, serum, and induced sputum. PLoS ONE 2012; 7: e46207.

35 McElvaney NG. Alpha-1 antitrypsin therapy in cystic fibrosis and the lung disease associated with alpha- 1 antitrypsin deficiency. Ann Am Thorac Soc 2016; 13 Suppl. 2: S191-S196.

36 Kolaczkowska E, Jenne CN, Surewaard BG, et al. Molecular mechanisms of NET formation and degradation revealed by intravital imaging in the liver vasculature. Nat Commun 2015; 6: 6673.

37 O'Donnell AE, Barker AF, Ilowite JS, et al. Treatment of idiopathic bronchiectasis with aerosolized recombinant human DNase I. rhDNase Study Group. Chest 1998; 113: 1329-1334.

38 Dubois AV, Gauthier A, Brea D, et al. Influence of DNA on the activities and inhibition of neutrophil serine proteases in cystic fibrosis sputum. Am J Respir Cell Mol Biol 2012; 47: 80-86.

39 Podolska MJ, Mahajan A, Hahn J, et al. Treatment with DNases rescues hidden neutrophil elastase from aggregated NETs. J Leukocyte Biol 2019; 106: 1359-1366. 